\title{
Vollständige Kassenkreditentschuldung kommunaler Gebietskörperschaften
}

\author{
Politische Zielsetzung und administrative Umsetzung der \\ HESSENKASSE
}

\author{
Thomas Duve/Kerstin Kümpel
}

Die Kassenkreditverschuldung der hessischen Kommunen hatte sich in der zurückliegenden Dekade drastisch erhöht und bei vielen Kommunen eine nachhaltige, generationengerechte Haushaltsführung stark gefährdet. Durch ein umfassendes Entschuldungsprogramm (HESSENKASSE) wurde insbesondere mit Blick auf die stets drohenden zukünftigen Zinsänderungsrisiken eine vollständige Rückführung der Kassenkredite erreicht. Im Rahmen dieses Programms hat das Land im Sinne einer „Hilfe zur Selbsthilfe“ den Kommunen sämtliche Kassenkredite abgenommen und die betroffenen Kommunen zu verlässlichen und zumutbaren Konditionen an der stetigen Tilgung beteiligt. In diesem Aufsatz wird die administrative Umsetzung der HESSENKASSE im Detail erläutert. Darüber hinaus werden auch die Maßnahmen benannt, die einen erneuten Aufwuchs der Kassenkredite eindämmen sollen. Abschließend werden Faktoren herausgearbeitet, die sich im Lichte der gewonnenen praktischen Erfahrungen als besonders erfolgsversprechend für die Umsetzung eines so umfassenden Entschuldungsprogramms erwiesen haben und auch eine Orientierung für andere Flächenländer sein können.

\section{Die Verschuldungssituation in Hessen vor der HESSENKASSE}

Die kommunale Verschuldung durch Kassenkredite ist in Deutschland in den letzten 25 Jahren regelrecht explosionsartig angestiegen. Mit Blick auf die Kassenkredite des öffentlichen Gesamthaushaltes

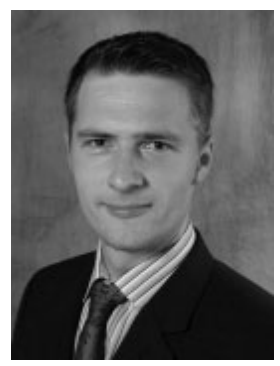

Dr. Thomas Duve

Referent im Hessischen Ministerium der Finanzen. haben sich die Kassenkredite der Kommunen bundesweit von 1992 bis 2017 nahezu verdreißigfacht. ${ }^{1}$ Dieser massive Anstieg ist jedoch keinesfalls ein bundesweit flächendeckendes Phänomen, welches alle Flächenländer gleichermaßen betrifft. Vielmehr sind deutliche Unterschiede zwischen den Ländern erkennbar. Das Land

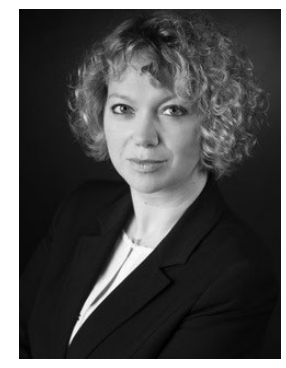

Kerstin Kümpel

Referentin im Hessischen Ministerium der Finanzen.
Hessen zählte bis 2017 regelmäßig zu den vier Ländern, in denen die Kassenkredite der Kommunen besonders hohe Stände erreichten. $^{2}$

In Hessen hatte die Kassenkreditverschuldung im Jahr 2012 mit rd. 7,5 Mrd. Euro ihren absoluten Höhepunkt erreicht (vgl. Abb. 1).

Die besonders starken Anstiege in den beiden zurückliegenden Dekaden gingen insbesondere mit zwei negativen wirtschaftlichen Impulsen einher. Zum einen führte die wirtschaftliche Entwicklung im Umfeld der Rezession $2003^{3}$ auch bei den Kommunen zu hohen Finanzierungsdefiziten, was im Ergebnis bereits zu einer Verdreifachung der Kassenkreditbestände führte. Zum anderen hatte die Finanzund Wirtschaftskrise (2008/2009) erhebliche Auswirkungen auf die Finanzsituation der Kommunen. Die Kassenkredite waren dadurch innerhalb von nur vier Jahren von rd. 3,2 Mrd. Euro (2008) auf rd. 7,5 Mrd. Euro (2012) angestiegen. Begünstigt wurde dieser Anstieg in Hessen u.a. da-

Im vorliegenden Beitrag vertreten die Autoren ausschließlich ihre eigenen Ansichten.

1 Vgl. Statistisches Bundesamt: Schulden des Öffentlichen Gesamthaushalts, Fachserie 14 Reihe 5.

2 Bundesministerium der Finanzen: Eckdaten zur Entwicklung und Struktur der Kommunalfinanzen 2009-2018, S. 26 f., https://www. bundesfinanzministerium.de/Web/DE/ Themen/Oeffentliche_Finanzen/Foederale Finanzbeziehungen/Kommunalfinanzen/kommunalfinanzen.html (og.10.2019)

3 Vgl. Räth (2009), S. 204f. 


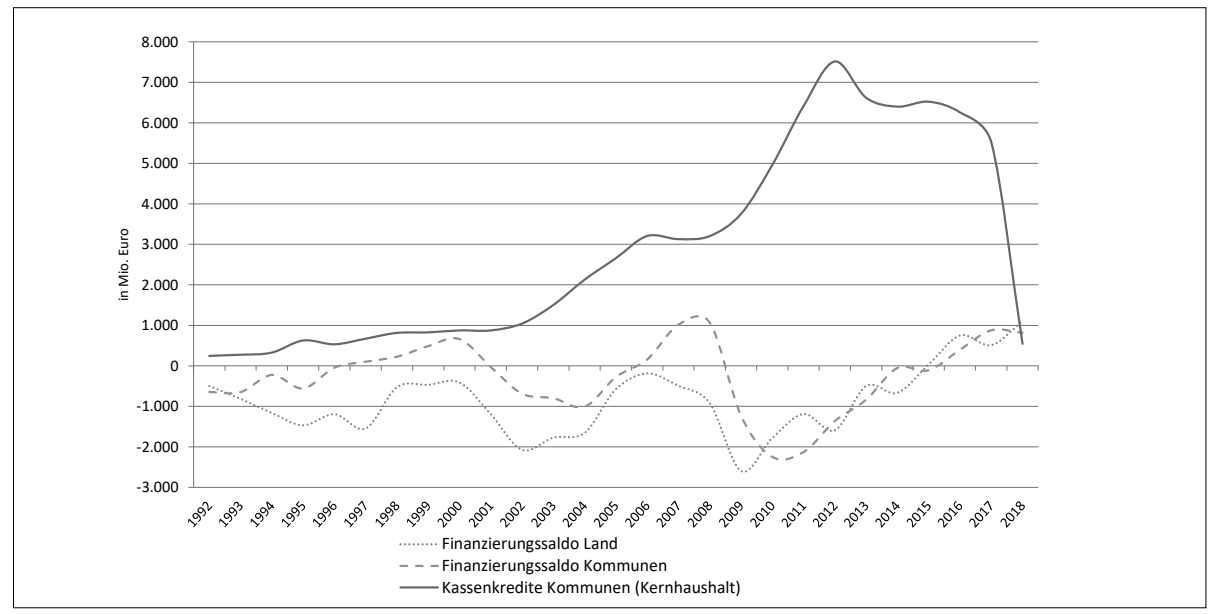

Abb. 1: Entwicklung der Finanzierungssalden der hessischen Kommunen und des Landes Hessen sowie der Kassenkreditverschuldung der Kommunen (Kernhaushalte)

Quellen: Hessisches Statistisches Landesamt, Jährliche Schuldenstandstatistik der Gemeinden und Gemeindeverbände, Rechnungsergebnisse der Gemeinden und Gemeindeverbände 1995-2017, Vierteljährliche Kassenergebnisse der Gemeinden und Gemeindeverbände 2018; Finanzierungssalden Land: HMdF; eigene Darstellung

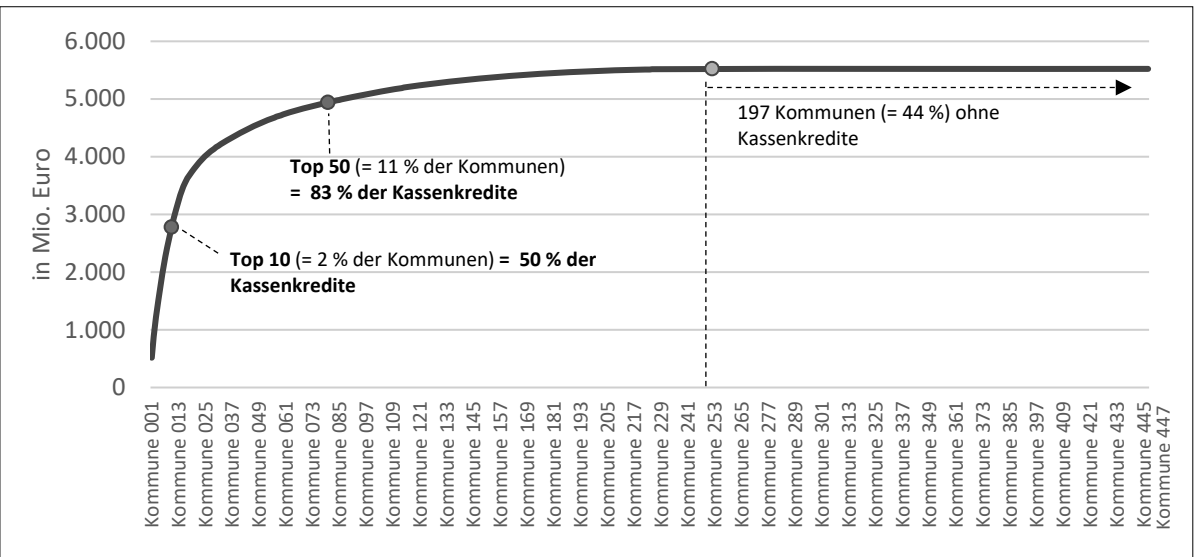

Abb. 2: Kumulierte Betrachtung der Kassenkreditverschuldung der hessischen Gemeinden und Gemeindeverbände 2017

Quelle: Hessisches Statistisches Landesamt, Jährliche Schulden der Gemeinden und Gemeindeverbände 2017; eigene Darstellung

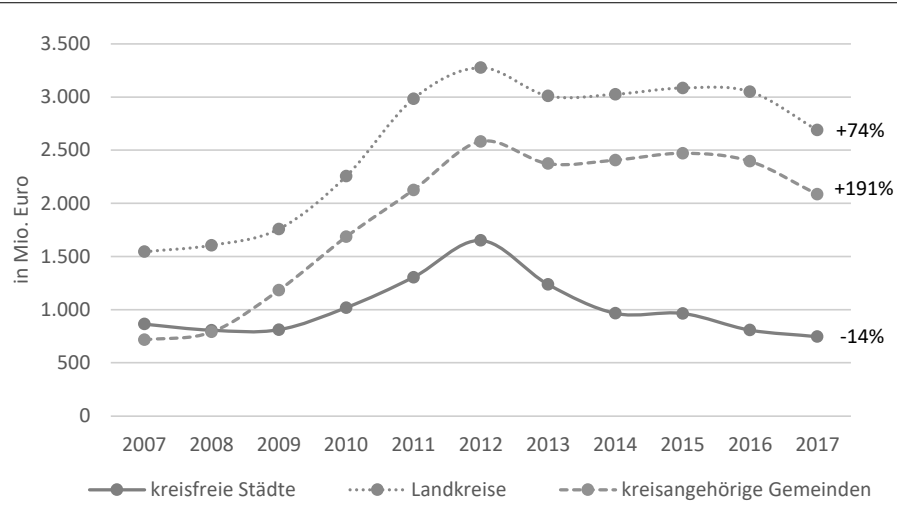

Abb. 3: Entwicklung der Kassenkreditverschuldung in Hessen nach kommunalen Gruppen Ouelle: Hessisches Statistisches Landesamt, Jährliche Schulden der Gemeinden und Gemeindeverbände 2007-2017; eigene Darstellung

durch, dass bis 2011 der Höchstbetrag für Kassenkredite nicht von der Kommunalaufsicht genehmigt werden musste. ${ }^{4}$ Zwar wurde eine Genehmigungspflicht für Kassenkredite durch eine entsprechende Änderung der Hessischen Gemeindeordnung ab dem Jahr 2012 wieder eingeführt ${ }^{5}$, doch hatten die Kassenkredite bis dahin bereits ein erhebliches Volumen angenommen, welches bei vielen Kommunen eine nachhaltige Haushaltsführung gefährdete. Eine Befreiung aus dieser Lage war vielen
Kommunen aus eigener Kraft zu diesem Zeitpunkt kaum möglich. Wie auch das Land selbst, verzeichneten viele Kommunen hohe Finanzierungsdefizite, was ihre Handlungsmöglichkeiten deutlich einschränkte.

Ein erster großer Schritt zur nachhaltigen Reduzierung der Kassenkreditbestände mit Hilfe des Landes war die Umsetzung des Kommunalen Schutzschirms, über den bei 100 hessischen Kommunen Schulden in Höhe von rd. 2,8 Mrd. Euro (davon rd. 1,8 Mrd. Euro Kassenkredite) abgelöst wurden. ${ }^{6}$ Diese Hilfe zur Selbsthilfe war verbunden mit nachweislichen Konsolidierungsbemühungen seitens der Kommunen. Durch die gemeinsamen Anstrengungen konnten sich die hessischen Kommunen in Folge dieses Entschuldungsprogramms von den Höchstständen entfernen. Trotz des anhaltenden Rückgangs der Kassenkredite, der u.a. vor dem Hintergrund der insgesamt guten wirtschaftlichen Lage nicht nur anzunehmen, sondern auch erforderlich war, verblieb letztlich noch ein relativ hoher Betrag in den kommunalen Haushalten. Im Jahr 2017 belief sich dieser Betrag auf rd. 5,5 Mrd. Euro.

Nicht unerheblich war dabei die Tatsache, dass dieser Kassenkreditbestand nicht auf alle $447^{7}$ hessischen Kommunen entfiel. Zum Jahresende 2017 waren 197 Kommunen kassenkreditfrei (vgl. Abb. 2). Die zehn Kommunen mit der höchsten absoluten Verschuldung machten bereits rd. 50 Prozent der gesamten Kassenkreditverschuldung in Hessen aus.

Unterschiede lassen sich sowohl in der Höhe als auch in der Entwicklung der Kassenkredite in einer differenzierten Betrachtung nach kommunalen Gruppen $^{8}$ erkennen (vgl. Abb. 3). Hinsichtlich der absoluten Beträge verzeichneten die

4 Vgl. Herrmann (2011), S. 688.

5 Vgl. Gesetzentwurf der Fraktionen der CDU und der FDP für ein Gesetz zur Änderung der Hessischen Gemeindeordnung und anderer Gesetze, LT-Drs. 18/4031 vom 10. Mai 2011.

6 Vgl. Keilmann/Duve/Gnädinger (2013), S. $115 \mathrm{ff}$.

7 Seit dem Jahr 2018 liegt die Zahl der Kommunen aufgrund einer Gemeindefusion bei 444 .

8 In Hessen gibt es mit Gebietsstand zum 31. Dezember 201721 Landkreise, fünf kreisfreie Städte und 421 kreisangehörige Gemeinden. 
Landkreise dauerhaft die höchsten Kassenkreditbestände. Der höchste Zuwachs von 2007 bis 2017 entfiel auf die kreisangehörigen Gemeinden, bei denen sich die Kassenkredite nahezu verdreifacht hatten. Ein leichter Rückgang der Kassenkreditbestände war hingegen bei den kreisfreien Städten zu verzeichnen. Dies darf jedoch nicht darüber hinwegtäuschen, dass die kreisfreien Städte die höchste Pro-KopfVerschuldung durch Kassenkredite aufwiesen.

Insgesamt verdeutlichen diese Daten nicht nur die in Hessen bestehende erhebliche Heterogenität in der Kassenkreditverschuldung, sondern ebenso auch die damit einhergehenden besonderen Anforderungen an ein Entschuldungsprogramm.

\section{Zielsetzung und Finanzierung der HESSENKASSE}

Aufgrund der bereits seit mehreren Jahren anhaltenden Niedrigzinsphase wirken sich die Zinsbelastungen durch aufgenommene Kassenkredite in den kommunalen Haushalten gegenwärtig nur relativ geringfügig auf der Aufwandsseite aus. Dies mochte den Handlungsdruck subjektiv mindern. Gleichwohl waren im Jahr 2017 die Kassenkreditbestände wie bereits ausgeführt noch immer bedenklich hoch. Im Falle eines erneuten Zinsanstiegs hätten den kommunalen Haushalten erhebliche Mehrbelastungen gedroht und die finanzielle Leistungsfähigkeit wäre massiv gefährdet gewesen. Zudem schien zum damaligen Zeitpunkt das Zinsniveau einen Tiefpunkt erreicht zu haben. ${ }^{9}$ Ein möglicher Wiederanstieg der Zinsen hätte die durch die Konsolidierungsbemühungen der letzten Jahre geschaffenen finanziellen Freiräume durch anwachsende Zinsbelastungen sukzessive verdrängt. Ein Anstieg des Zinsniveaus um lediglich 0,1 Prozentpunkte hätte 2017 zu einer Erhöhung der Zinsbelastung um rund 5 Mio. Euro geführt. Der Handlungsdruck war vor dem Hintergrund einer ungewissen zukünftigen Zinsentwicklung demnach objektiv vorhanden. Um langfristig diese Risiken zu minimieren, bedurfte es daher einer vollständigen Rückführung der noch vorhandenen Kassenkredite.

Das dafür geschaffene Landesprogramm mit dem Namen HESSENKASSE hat das strategische Ziel, das geschilderte Zinsänderungsrisiko und die damit drohenden Mehrbelastungen für die kommunalen Haushalte durch eine konsequente Rückführung der Schulden drastisch $\mathrm{zu}$ reduzieren sowie einen Wiederaufwuchs der Kassenkredite nachhaltig $\mathrm{zu}$ vermeiden. Das operative Ziel der HESSENKASSE lag in der Übernahme

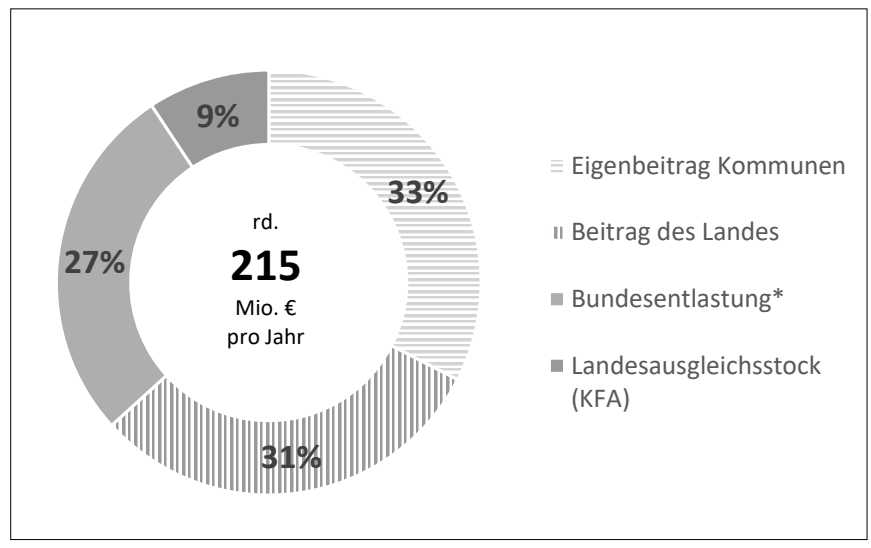

Abb. 4: Finanzierung der Hessenkasse

Quelle: Hessisches Ministerium der Finanzen; eigene Darstellung ${ }^{*}$ Mittel vom Bund, die dem Land zur Entlastung der Kommunalhaushalte zur Verfügung gestellt werden.

der Kassenkredite durch das Land und die Organisation einer stetigen Tilgung sowohl durch das Land als auch durch die Kommunen zu angemessenen Konditionen.

Im Rahmen der HESSENKASSE ${ }^{10}$ organisierte das Land für die Kommunen auf Antrag eine Ablösung der Kassenkredite des Kernhaushalts bei deren Gläubigerkreditinstituten. Dabei bediente sich das Land der Wirtschafts- und Infrastrukturbank Hessen (WIBank), die die Kredite bei den Kommunen übernahm und sich dabei am Kapitalmarkt refinanzierte.

Die Teilnahme an der HESSENKASSE war grundsätzlich freiwillig. Insgesamt wurden durch die HESSENKASSE Kassenkredite i.H.v. rd. 4,9 Mrd. Euro abgelöst. Zum Zeitpunkt der konzeptionellen Erarbeitung des Programms, im Jahr 2017, wurde noch von einem deutlich höheren Betrag ausgegangen, da ausschließlich auf statistische Daten zurückgegriffen werden konnte. Der Kassenkreditbestand lag zum 31. Dezember 2016 noch bei rd. 6,25 Mrd. Euro.

Über die Kassenkreditentschuldung hinaus wurde seitens des Landes ein Investitionsprogramm eingerichtet und in die HESSENKASSE integriert. ${ }^{11}$ Im Rahmen dieses Programms sollten finanz- oder strukturschwache Kommunen, die ihre stetige Zahlungsfähigkeit nachweislich ohne den Aufbau von Kassenkreditbeständen sicherstellen konnten, auf Antrag eine Investitionsförderung erhalten. Diese Förderung beläuft sich auf insgesamt rd. 627 Mio. Euro. Auf diese Weise sollte den Kommunen geholfen werden, die es trotz geringer Erträge in der Vergangenheit geschafft haben, ihre Aufwendungen ohne eine dauerhafte Finanzierung durch Kassenkredite zu decken. Diese Kommunen sollten in die Lage versetzt werden, etwaige unterlassene Investitionen oder Instandhaltungen nachzuholen. ${ }^{12}$

Zur Finanzierung der HESSENKASSE wurde das Sondervermögen „Hessenkasse" errichtet, welches neben der Finanzierung der Kassenkreditentschuldung auch zur Förderung kommunaler und kommunalersetzender Investitionen verwendet wird. Über einen Zeitraum von 30 Jahren entsteht im Sondervermögen für die Zinsund Tilgungszahlungen ein durchschnittlicher jährlicher Finanzierungsbedarf von rd. 215 Mio. Euro (vgl. Abb. 4). Für den gesamten Finanzierungszeitraum von 30 Jahre wurde ein Zinssatz von 1,523 Prozent ermittelt, wodurch ein planbarer kontinuierlicher Zahlungsplan erreicht werden konnte.

Das Sondervermögen finanziert sich dabei aus Mitteln des Landeshaushalts

9 Vgl. Brand/Steinbrecher (2018), S. 1.

10 Gesetz zur Sicherstellung der finanziellen Leistungsfähigkeit der hessischen Kommunen bei liquiditätswirksamen Vorgängen und zur Förderung von Investitionen (HessenkasseG) vom 25. April 2018 (GVBI. S. 59-67).

11 Vgl. $\$ 6$ HessenkasseG

12 Vgl. Gesetzentwurf der Fraktionen der CDU und BÜNDNIS 90/DIE GRÜNEN für ein Gesetz zur Sicherstellung der finanziellen Leistungsfähigkeit der hessischen Kommunen bei liquiditätswirksamen Vorgängen und zur Förderung von Investitionen (HessenkasseG), LT-Drs. 19/5957 vom 23. Januar 2018, S. 14. 


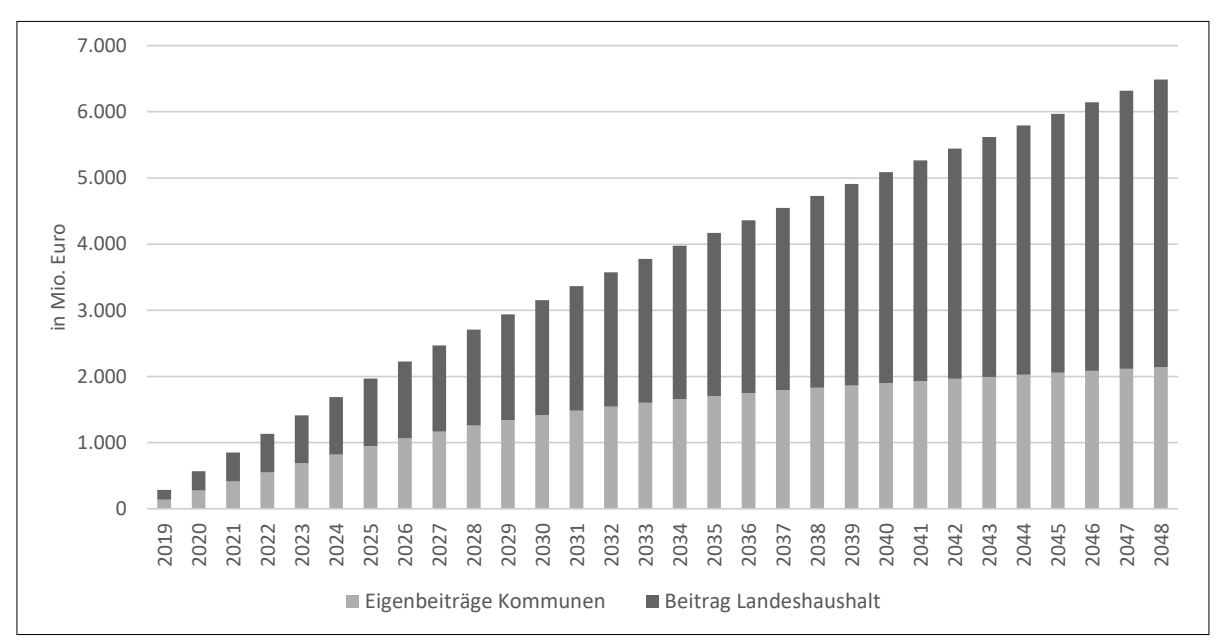

Abb. 5: Kumulierte Darstellung der Zins- und Tilgungszahlungen für die gesamte Laufzeit der HESSENKASSE

Quelle: HMdF, eigene Darstellung

und aus Eigenbeiträgen der am Entschuldungsprogramm teilnehmenden Kommunen. Während der Anteil aus Mitteln, die über den Landeshaushalt zur Verfügung gestellt werden, jährlich 145 Mio. Euro beträgt, sind von den Kommunen direkt im Durchschnitt der Laufzeit rd. 70 Mio. Euro pro Jahr als Eigenbeitrag aufzubringen.

Die Eigenbeiträge der betroffenen Kommunen, die in das Sondervermögen eingezahlt werden, belaufen sich pauschal auf 25 Euro je Einwohner. Mit den Eigenbeiträgen tilgt jede Kommune die Hälfte ihrer Kassenkredite. Diese Beitragszahlungen erfolgen - in Abhängigkeit von der individuellen Kassenkreditverschuldung der jeweiligen Kommune - über einen individuell ermittelten Zeitraum, maximal jedoch bis zu 30 Jahre.

Mit Sicht auf die volle Laufzeit des Entschuldungsprogramms ergibt sich ein Gesamtfinanzierungsbedarf i.H.v. rd. 6,5 Mrd. Euro, welcher über die oben genannten durchschnittlichen jährlichen Finanzierungsbeiträge $\mathrm{zu}$ decken ist (vgl. Abb. 5). Dies verdeutlicht, dass dem Land und den Kommunen noch ein langer Weg bevorsteht und in den folgenden Dekaden jährlich Mittel in den Haushalten von Land und Kommunen zur Abfinanzierung der Kassenkreditbestände gebunden sind.

Da sich in jeder Kommunen kurzfristig die finanziellen Rahmenbedingungen verändern können, erschien ein unabdingbares Festhalten an der jährlichen Zahlung des Eigenbeitrags über Jahre hinweg als wenig zweckdienlich. Durch die geschaffene Möglichkeit, sowohl Ratenpausen als auch Sondertilgungen bezüglich der Beitragszahlungen zu beantragen, sollen die Kommunen in den Folgejahren flexibel auf ihre individuelle Finanzsituation reagieren können.

Zur Vereinfachung des Verwaltungsverfahrens werden die Eigenbeiträge mit Zahlungen des Landes an die Kommunen (Gemeindeanteile an der Einkommensteuer) verrechnet. Bei den Landkreisen, bei denen eine solche Verrechnung nicht möglich ist, werden die Eigenbeiträge mit den Schlüsselzuweisungen aus dem Kommunalen Finanzausgleich verrechnet.

\section{Administrative Umsetzung und Ergebnis der HESSENKASSE}

Hinsichtlich der Bestimmung der individuellen Kassenkreditbestände, die im Jahr $2018 \mathrm{zu}$ erwarten waren und durch die HESSENKASSE abgelöst werden sollten, genügte die alleinige Heranziehung der Schuldenstatistik nicht aus. So lässt sich den statistischen Daten nicht entnehmen, in welchem Umfang Kassenkredite enthalten sind, die zur Vorfinanzierung von Investitionen oder öffentlich-rechtlicher Forderungen dienen, nicht zur Sicherstellung der Zahlungsfähigkeit der Kommune erforderlich sind oder ggf. aufgrund der finanziellen Leistungsfähigkeit der werden können. ${ }^{13}$ Zudem konnte anhand der Statistik (Stand 31. Dezember 2016) kein Rückschluss auf den zu erwartenden Kassenkreditbestand im Jahr 2018 gezoKommune eigenständig zurückgeführt gen werden, der letztlich auch aufgrund der guten wirtschaftlichen Gesamtlage und der gegenwärtig vorliegenden Finanzierungsüberschüsse tendenziell rückläufig sein musste. Es bedurfte daher eines zweckdienlichen Verfahrens zur Ermittlung der tatsächlich im Jahr 2018 relevanten Ablösungsbeträge.

Vor diesem Hintergrund erfolgte die Umsetzung der HESSENKASSE in zwei Schritten. In einem ersten Schritt wurden die aktuellen Kassenkreditbestände anhand von Fragebögen bei allen hessischen Kommunen im September 2017 erfasst. Im Rahmen dieser Abfrage wurden u.a. erfasst, in welchem Umfang in den aktuellen Kassenkreditbeständen Kredite zur Vorfinanzierungen von Investitionen enthalten waren, Kredite an Eigenbetriebe weitergereicht wurden und in welchem Umfang den Kassenkrediten liquide Mittel gegenüberstanden. Anhand dieser Daten, die seitens des Finanzministeriums, des Innenministeriums, der Regierungspräsidien (Kommunalaufsicht) und der WIBank ausgewertet wurden, konnten bereits erste Einschätzungen erfolgen, ob die jeweilige Kommune für das Entschuldungsprogramm oder das Investitionsprogramm infrage kommt und mit welcher Entschuldungshöhe zu rechnen ist. Im Zuge der Ermittlung der Entschuldungshöhe spielten die individuellen Ursachen der Verschuldung und damit eine potenzielle Schuldfrage keine Rolle, da dies für das übergeordnete Ziel des Entschuldungsprogramms unerheblich war.

Diese gewonnenen Informationen genügten jedoch noch nicht, um konkrete Ablösungsbeträge beziffern zu können. Zum einen ergaben sich mitunter Nachfragen aus den Angaben der Kommunen in den Fragebögen. Zum anderen musste auch ein „Blick nach vorn“ gewagt werden, der es ermöglicht, auf Basis der Haushaltsplanungen der Kommunen für das Jahr 2018 den voraussichtlichen ablösungsfähigen Kassenkreditbestand (Ablösungshöchstbetrag) jeder einzelnen Kommunen zu ermitteln. Da dies nur durch Individualgespräche mit den Kommunen erzielt werden konnte, wurde allen Kom-

13 Vgl. §1 Abs. 1 HessenkasseG. 


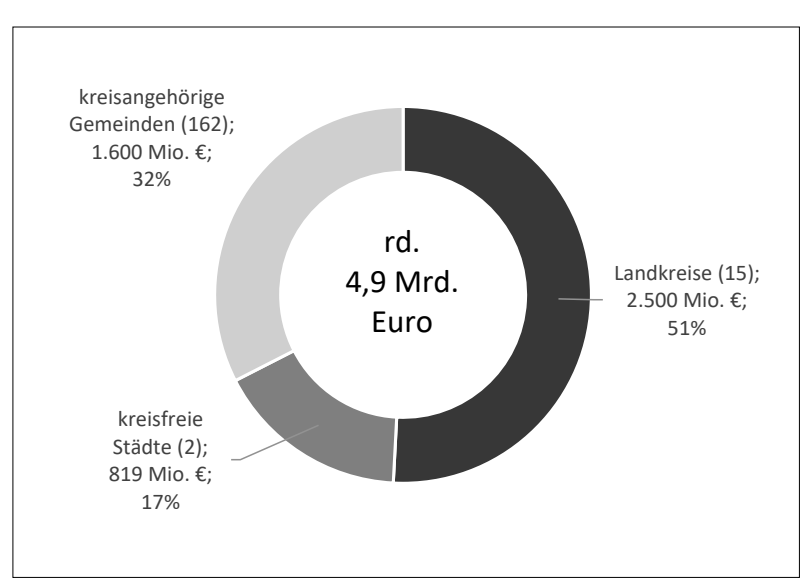

Abb. 6: Verteilung der Kassenkreditentschuldung nach kommunalen Gruppen (inkl. Anzahl der Kommunen)

Quelle: Hessisches Ministerium der Finanzen; eigene Darstellung

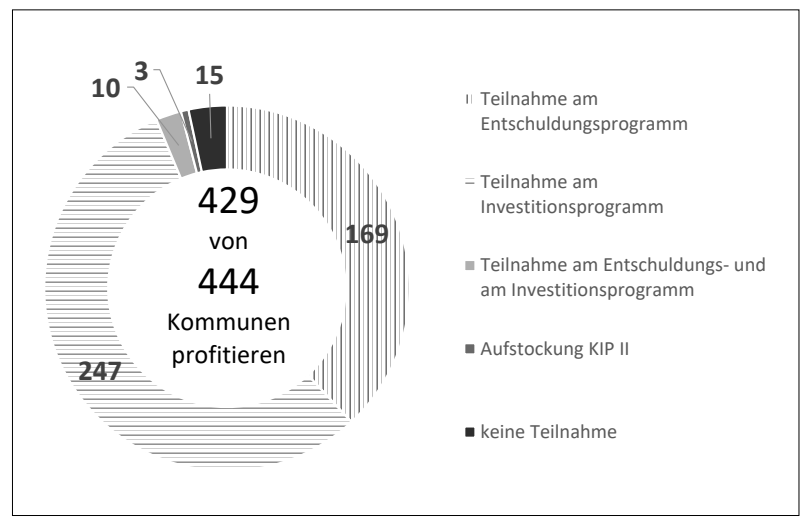

Abb. 7: Anzahl der durch die Hessenkasse profitierenden Kommunen

Quelle: Hessisches Ministerium der Finanzen; eigene Darstellung

munen, die zunächst für das Entschuldungsprogramm infrage kamen, zu Erörterungsgesprächen ${ }^{14}$ in das Finanzministerium eingeladen.

Insgesamt wurden zwischen November 2017 und Mai 2018 Gespräche mit 251 Kommunen (zuzüglich Zweitgespräche) geführt. Die Notwendigkeit zu diesen Gesprächen bestätigte sich schnell, denn nur auf diese Weise konnten die spezifischen Gegebenheiten in den einzelnen Kommunen erörtert und angemessen bei der Ermittlung der Ablösungsbeträge berücksichtigt werden. In einigen Fällen veränderten sich erst durch die Gespräche die Sachverhalte so sehr, dass Kommunen z.B. nicht mehr für das Entschuldungsprogramm, sondern - weil keine Kassenkredite zum voraussichtlichen Ablösungszeitpunkt vorgelegen hätten - nur noch für das Investitionsprogramm infrage kamen. Am Ende der protokollierten Gespräche stand fest, welche Kommunen am Entschuldungsprogramm und welche am Investitionsprogramm teilnehmen konnten und welche Kassenkreditbeträge ablösungsfähig sind. In einigen wenigen Fällen wurde auch eine gleichzeitige Teilnahme sowohl am Entschuldungsprogramm als auch - anteilig - am Investitionsprogramm zugelassen. Beschränkt wurde die Möglichkeit auf Kommunen, bei denen eine Teilnahme am Investitionsprogramm deutlich wirtschaftlicher erschien als eine reine Kassenkreditentschuldung, eine Rückführung der Kassenkredite aus eigener Kraft aber nachweislich durch die Kommune nicht geleistet werden konnte.

Bevor die Übernahme der Kassenkredite vollzogen werden konnte, musste jede Kommune eine Verpflichtungserklärung von der Mehrheit der Mitglieder der Gemeindevertretung bzw. des Kreistags beschließen. Dabei verpflichteten sich die Kommunen, neben der Zahlung des Eigenbeitrags in Höhe von 25 Euro je Einwohner, den Ergebnis- und Finanzhaushalt in Planung und Rechnung ab dem Jahr 2019 nach $\mathbb{S}$ 92 Abs. 4 bis 6 der Hessischen Gemeindeordnung auszugleichen sowie die Vorgaben zu den Liquiditätskrediten nach $\mathbb{S}$ 105 der Hessischen Gemeindeordnung zu beachten. ${ }^{15}$

Von den 444 hessischen Kommunen nahmen insgesamt 179 Kommunen ${ }^{16}$ am Entschuldungsprogramm teil (vgl. Abb. 6). Ihnen wurden noch im Jahr 2018 Kassenkredite i.H.v. rd. 4,9 Mrd. Euro abgenommen.

Nachdem die Kommunen Ablösungsvereinbarungen mit der WIBank geschlossen hatten, erfolgte die Ablösung der Kassenkredite durch die WIBank zu zwei Stichtagen, zum 17. September sowie zum 17. Dezember 2018. Die Kommunen hatten sicherzustellen, dass die Kassenkredite zum jeweiligen Stichtag ablösungsfähig waren. Auf diese Weise wurden 2,9 Mrd. Euro an Kassenkrediten direkt bei den Kommunen abgelöst. Bei längeren Laufzeiten der Kassenkreditverträge erfolgte die Ablösung der Kassenkredite in der Regel in Form eines Schuldnerwechsels. Insgesamt konnten dadurch bei Kassenkrediten in Höhe von 1,8 Mrd. Euro die Zahlung von Vorfälligkeitsentschädigungen und damit zusätzlichen Kosten vermieden werden. Sofern dies nicht möglich war, erfolgt an Stelle einer Kassenkreditablösung die Zahlung von Zinsdienst- und Entschuldungshilfen in Höhe von 200 Mio. Euro.

Die Mehrheit der Kommunen nimmt jedoch nicht das Entschuldungsprogramm, sondern das angebotene Investitionsprogramm in Anspruch. Die für das Investitionsprogramm antragsberechtigten Kommunen mussten nach der Antragstellung die Kassenkreditfreiheit zum Stichtag 30. Juni 2018 nachweisen, sofern kein davon abweichender Stichtag in der zweiten Jahreshälfte 2018 beantragt wurde. Daraufhin wurde der beantragte individuelle Investitionszuschuss und der von der Kommune zu erbringende Eigenanteil von einem Neuntel des Zuschusskontingents beschieden. Der Verwendungszweck für die Investitionsförderung war bewusst sehr weit gefasst worden, um die individuellen Investitionsbedarfe der Kommunen angemessen berücksichtigen zu können.

Lediglich 15 Kommunen nehmen nicht an der HESSENKASSE teil, da sie aufgrund ihrer besonders hohen Finanzkraft in beiden Programmenteilen nicht antragsberechtigt waren. Drei Schulträgerkommunen (zwei kreisfreie Städte und ein Landkreis) erhielten anstelle einer Teilnahme am Investitionsprogramm ein um insgesamt rd. 25 Mio. Euro erhöhtes Kontingent im Kommunalinvestitionsprogramm „KIP macht Schule!“ (KIP II). Im Investitionsprogramm der HESSENKASSE hätte diesen drei Kommunen andernfalls ledig-

14 An diesen Gesprächen nahmen Vertreter der jeweiligen Kommune, des Finanzministeriums, des Innenministeriums, der Regierungspräsidien, der unteren Kommunalaufsicht sowie der WIBank teil.

15 Vgl. § 2 Abs. 2 HessenkasseG

16 Davon nehmen zehn Kommunen zusätzlich auch am Investitionsprogramm der Hessenkasse teil. 
lich ein Mindestkontingent von 750.000 Euro zugestanden.

Die unterschiedliche Partizipation der Kommunen an der HESSENKASSE (vgl. Abb. 7) spiegelt die eingangs beschriebenen heterogenen Rahmenbedingungen in Hessen wider. Dieses Ergebnis reflektiert den Versuch, dieser Heterogenität angemessen und ausgewogen Rechnung zu tragen.

\section{Anpassung finanzaufsichtsrechtli- cher Regelungen}

Das enorme Volumen der Hessenkasse und der Finanzierungszeitraum von 30 der längerfristigen Finanzierung laufender Aufwendungen konsumtiv eingesetzt. Darüber hinaus wurde Kassenkredite auch zur Finanzierung der Tilgung investiver, ordentlicher Kredite aufgenommen. ${ }^{18}$

Flankierend zur HESSENKASSE wurden in Hessen daher Änderungen der Hessischen Gemeindeordnung (HGO) und der Gemeindehaushaltsverordnung (Gem$\mathrm{HVO}$ ) vorgenommen. ${ }^{19}$ Insbesondere geht es dabei um die Wahrung des ursprünglichen Zwecks des Kassenkredites als kurzfristiger Liquiditätskredit. Die Aufnahme von Liquiditätskrediten zur Überbrückung von Liquiditätsengpässen ist dabei weiterhin möglich. Zukünftig sollen Li-

\section{"Eine zweckentfremdete Verwendung von Kassenkrediten muss zukünftig zwingend vermieden werden."}

Jahren macht eines deutlich: Ein solches Entschuldungsprogramm ist in der Geschichte des Landes Hessen einmalig und muss auch einmalig bleiben.

Daher ist es unerlässlich, den erneuten Aufwuchs von Kassenkreditbeständen deutlich einzudämmen bzw. gänzlich zu vermeiden. Letztlich wird es dadurch auch nachfolgenden Generationen ermöglicht, die Kommunalpolitik aktiv auszugestalten, ohne ständig aufgrund erforderlicher Sparbemühungen zum bloßen Agieren verdammt zu sein.

Eine zweckentfremdete Verwendung von Kassenkrediten ${ }^{17}$ muss zukünftig zwingend vermieden werden, um einen nachhaltigen Erfolg gewährleisten $\mathrm{zu}$ können. In der Vergangenheit wurden Kassenkredite neben der kurzfristigen Liquiditätssicherung und zur zulässigen Vorfinanzierung von Investitionen auch zum nicht gesetzlich intendierten Zweck quiditätskredite jedoch nur noch als ein „Nothilfeinstrument" verstanden und in Anspruch genommen und nicht, wie in der Vergangenheit zum Teil praktiziert, als beliebig genutztes Zahlungsmittel verwendet werden.

Im Wesentlichen wurden folgende gesetzliche Änderungen ${ }^{20}$ in der HGO vorgenommen:

- Um negative Abweichungen im Haushaltsvollzug zu vermeiden, wird zukünftig neben der Planung auch die Rechnung den Anforderungen für den Haushaltsausgleich unterworfen $(\mathbb{S} 92$ Abs. 2 HGO).

- Die Kommunen müssen ab dem Haushaltsjahr 2019 zum Ausgleich des Finanzhaushaltes einen Zahlungsmittelüberschuss generieren, der in einer solchen Höhe anfällt, dass daraus sowohl Tilgungsauszahlungen als auch der kommunale Eigenbeitrag in Höhe von $25 €$ je Einwohner für das Sonder- vermögen „HESSENKASSE“ geleistet werden können ( $\mathbb{S} 92$ Abs. 5 Nr. 2 HGO).

- In die HGO wird ein ausdrückliches Überschuldungsverbot aufgenommen ( 92 Abs. 7 HGO).

- Die Regelungen zur Erstellung von Haushaltssicherungskonzepten werden erweitert. Unter anderem müssen zukünftig als unerlässlicher Mindeststandard im Haushaltssicherungskonzept konkrete, quantifizierbare Konsolidierungsmaßnahmen und einen Konsolidierungszeitraum benannt werden $(\mathbb{S}$ 92a HGO).

- Die Begrifflichkeit „Kassenkredit“ wird durch „Liquiditätskredit“ ersetzt ( $\mathbb{S} 94$ Abs. 2 Nr. 2, 105 HGO).

- Die Genehmigungstatbestände werden erweitert. Sowohl eine Abweichung vom Gebot des Haushaltsausgleichs nach $\mathbb{S} 92$ Abs. 5 HGO als auch das Haushaltssicherungskonzept bedürfen der Genehmigung der Aufsichtsbehörde ( $\int 97 a$ Nr. 1 und Nr. 2 HGO).

- Die Anforderungen zur Aufnahme und Rückführung von Liquiditätskrediten werden erweitert. Unter anderem ist der Höchstbetrag durch eine Liquiditätsplanung zu dokumentieren. In dieser Planung muss den Aufsichtsbehörden dargelegt werden, warum der von der Kommune festgesetzte Höchstbetrag erforderlich ist. Zudem sollen Liquiditätskredite spätestens bis zum Ende des Haushaltsjahres zurückgeführt werden. Eine Abweichung von dieser Rückführungsfrist ist nur in Ausnahmefällen erlaubt. (\$ 105 HGO).

- Zur Vermeidung eines sofortigen Rückgriffs auf Liquiditätskredite soll eine Liquiditätsreserve gebildet werden. Der Bestand an flüssigen Mitteln soll sich dabei in der Regel auf mindestens zwei Prozent der Summe der Auszahlungen auslaufender Verwaltungstätigkeit nach dem Durchschnitt der drei dem Haushaltsjahr vorangehenden Jahre belaufen ( $\mathbb{1 0 6}$ Abs. 1 HGO).

$17 \mathrm{Vgl}$. Gröpl/Heinemann/Kalb (2010), S. 178ff.

18 Vgl. Gesetzentwurf zum HessenkasseG, a.a.O, S. $22 \mathrm{f}$.

19 Vgl. Artikel 4 und 5 HessenkasseG.

20 Vgl. Gesetzentwurf zum HessenkasseG, a.a.O., S. $23 \mathrm{ff}$.

21 Vgl. hierzu u.a. Boettcher (2012), S. $77 \mathrm{ff}$. 
- Die Pflicht zur fristgerechten Aufstellung des Jahresabschlusses wird mit der Haushaltsgenehmigung verknüpft ( $(112$ Abs. 10 HGO).

\section{Erfolgsfaktoren für ein wirksames Entschuldungsprogramm}

Mit der praktischen Umsetzung der HESSENKASSE gehen zahlreiche Erfahrungen einher. Daraus lassen sich verschiedene Faktoren $^{21}$ ableiten, die maßgeblich die Wirksamkeit und damit den Erfolg eines solchen Entschuldungsprogramms beeinflussen können.

\section{Anreizwirkung und Nachhaltigkeit}

Zunächst ist der Umfang eines solchen Programms relevant. Ein zu geringes Volumen kann wie ein „Tropfen auf dem heißen Stein“ wirken und letztlich langfristig zu keinen nennenswerten Erfolgen führen. Die ausschließliche Gewährung von Zinsdiensthilfen entlastet zwar die Kommunen, lässt die Kommunen jedoch mit der eigentlichen Herausforderung, der Rückführung der Kassenkredite, allein. Da die Kassenkreditverschuldung in vielen Kommunen ein Volumen angenommen hat, welches auch auf Jahrzehnte hin nicht aus eigener Kraft zurückgeführt werden kann, ist ein signifikantes Ablösungsvolumen zielführend, um das Problem nachhaltig zu lösen. In Hessen ist die Entscheidung zugunsten einer vollständigen Ablösung der Kassenkredite gefallen.

Ein reines „Bail-out“ durch das Land sollte aus ordnungspolitischen Gründen vermieden werden, um keine Fehlanreize zu setzen. ${ }^{22}$ Daher sollten die Kommunen an der kontinuierlichen Rückführung der eigenen Kassenkredite in jedem Fall in einem zumutbaren Umfang beteiligt werden. Gleichzeitig dürfen die Konditionen zu keiner Überlastung der Kommunen führen. Individualgespräche mit den Kommunen helfen dabei, die genauen Ablösungsbeträge zu ermitteln und die Konditionen im Detail zu erörtern. In Hessen wurden mit allen Kommunen, die am Entschuldungsprogramm teilnehmen, Gespräche geführt. Jede Kommune wird direkt an der Tilgung ihrer eigenen Kassenkredite beteiligt. Sie zahlt einen Eigenbeitrag i.H.v. von 25 Euro je Einwohner solange, bis sie damit die Hälfte ihrer abgelösten
Kassenkredite getilgt hat, längstens jedoch über einen Zeitraum von 30 Jahren.

Des Weiteren ist es zwingend erforderlich, geeignete Maßnahmen zu ergreifen, die einen erneuten Aufwuchs von Kassenkrediten vermeiden. Ohne eine Anpassung der finanzaufsichtlichen Rahmenbedingungen besteht die Gefahr, dass die Kommunen schnell in alte Muster verfallen. Im Ergebnis würden in den Kommunalhaushalten erneut erhebliche Beträge für die Tilgung und Zinszahlungen gebunden und die Handlungsspielräume für wachstumsfördernde Investitionen dagegen deutlich eingeschränkt werden. In Hessen wurden dazu Änderungen in der Gemeindeord- maßnahmen, wie z.B. die Anhebung von Realsteuerhebesätzen, zugelassen hat. Um ein solches Gefühl der Ungerechtigkeit zu vermeiden, bietet es sich an, diese Kommunen ebenfalls in geeigneter Weise zu unterstützen. In Hessen wurde kassenkreditfreien Kommunen die Teilnahme an einem Investitionsprogramm, welches Bestandteil der HESSENKASSE ist, ermöglicht.

Insgesamt erscheint eine flächendeckende, undifferenzierte Begünstigung aller Kommunen, ob durch ein Entschuldungsprogramm oder ein flankierendes Investitionsprogramm, nicht sachgerecht. Besonders steuerertragsstarke Kommu-

\section{"Die Kommunen sollten an der Rückführung ihrer eigenen Kassenkredite in jedem Fall in einem zumutbaren Umfang beteiligt werden."}

nung des Gemeindehaushaltsrechts vorgenommen, um Kassenkredite auf ihren ursprünglichen Zweck - die Überbrückung von kurzfristigen Liquiditätsengpässen zurückzuführen

\section{Gerechtigkeit und Akzeptanz}

Da bekanntlich nicht alle Kommunen innerhalb eines Flächenlandes hohe Kassenkreditbestände aufweisen, gehen diese Kommunen im Falle eines Entschuldungsprogramms „leer“ aus. Da es sich bei den kassenkreditfreien Kommunen nicht ausschließlich um steuerertragsstarke Kommunen handelt, kann bei diesen Kommunen durchaus ein Gefühl der Ungerechtigkeit entstehen und damit einen gewissen latenten Unfrieden innerhalb der kommunalen Familie verursachen. Schließlich haben sich oft genau diese Kommunen in der Vergangenheit nur das geleistet, was ihre finanzielle Leistungsfähigkeit auch unter Umsetzung von Konsolidierungs- nen können ihren Einwohnern deutliche höhere Standards bieten. Diesen Kommunen „ohne Not“ zusätzliche Mittel zur Verfügung zu stellen, die von steuerertragsschwächeren Kommunen wesentlich dringender benötigt werden, wäre kaum vermittelbar. Stattdessen sollten klar abgrenzbare Kriterien für die Teilnahme an der Kassenkreditentschuldung erarbeitet werden, die genau diesen Konflikt berücksichtigen. In Hessen wurden daher besonders steuerertragsstarke Kommunen von der HESSENKASSE ausgeschlossen. Die Hürden für diesen Ausschluss wurden jedoch sehr hoch gesetzt, so dass letztlich nur die Kommunen nicht teilnehmen konnten, die im Kommunalen Finanzausgleich im Zeitraum von 2004 bis 2018 mehr als zehnmal abundant waren.

22 Vgl. Heppke-Falk/Wolff (2007), S. $16 f$. 
Für die Akzeptanz eines Entschuldungsprogramms spielt auch der Verwaltungsaufwand und die mitunter gebotene Flexibilität in der Praxis eine wesentliche Rolle. In Hessen wurde unter diesen Gesichtspunkten beschlossen, den Eigenbeitrag der Kommunen bürokratiearm mit Zahlungen des Landes zu verrechnen, was auf beiden Seiten den Aufwand niedrig hält. Zudem wird den Kommunen ermöglicht, durch Ratenpausen oder Sondertilgungen flexibel auf aktuelle veränderte Rahmenbedingungen vor Ort zu reagieren.

Auch die Einbindung der beteiligten Akteure ist ein Aspekt, der die Akzeptanz gramms auswirken kann. In wirtschaftlich guten Zeiten können die erzielten Überschüsse teilweise auch für einen zusätzlichen Abbau der Kassenkreditverschuldung eingesetzt werden. Natürlich können sich diese konjunkturellen Rahmenbedingungen wieder in den Folgejahren ändern, was die Aufbringung der Tilgungsleistungen wieder erschweren dürfte. Gleichwohl ist es erforderlich, einen geeigneten Startpunkt zu finden und in wirtschaftlich guten Zeiten sind die finanziellen Spielräume in der Regel nicht nur größer, sondern erleichtern auch die Diskussionen und Entscheidungen auf allen Ebenen zugunsten eines Entschuldungsprogramms. In Hessen erzielen sowohl das Land als auch

\section{"Die Kommunalen Spitzenverbände waren Initiator der Idee einer möglichen Kassenkreditentschuldung und haben an der Entwicklung der HESSENKASSE aktiv mitgewirkt."}

eines Entschuldungsprogramms positiv beeinflussen kann. In Hessen hat das Finanzministerium als Bewilligungsstelle die Finanzaufsicht in Form des Innenministeriums und auch Vertreter der Kommunen am Entwicklungsprozess beteiligt. Konkret waren die Kommunalen Spitzenverbände nicht nur Initiator der Idee einer möglichen Kassenkreditentschuldung. Vielmehr haben sie auch im Rahmen einer Facharbeitsgruppe an der Entwicklung der HESSENKASSE aktiv mitgewirkt. Darüber hinaus wurde den Kommunen das Konzept der HESSENKASSE auf Regionalkonferenzen durch den Finanzminister vorgestellt.

\section{Gesamtwirtschaftliche Rahmenbedingungen}

Der Zeitpunkt eines Entschuldungsprogramms ist ein weiterer Faktor, der sich auf den langfristigen Erfolg des Pro-
Neben der guten wirtschaftlichen Gesamtsituation und den niedrigen Zinsen wirken sich auch Bundesentlastungen begünstigend auf das Entschuldungsprogramm aus. In Hessen werden finanzielle Mittel, die der Bund den Ländern über den Länderanteil an der Umsatzsteuer zur Entlastung der Kommunalhaushalte zur Verfügung stellt, zur anteiligen Finanzierung des Entschuldungsprogramms verwendet.

\section{Fazit und Ausblick}

Nur mit dem konsequenten und systematischen Abbau der aufgelaufenen Kassenkreditbestände bei gleichzeitiger Vermeidung eines Aufwuchses neuer Kassenkredite kann das Ziel erreicht werden, das den Kassenkrediten anhaftende Zinsänderungsrisiko deutlich zu reduzieren und damit die Finanzlage der Kommunen nachhaltig zu stärken. Mit der HESSENKASSE hat das Land Hessen unter Beachtung der heterogenen kommunalen Rahmenbedingungen ein facettenreiches Programm umgesetzt. Das operative Ziel, die Ablösung aller Kassenkredite wurde bereits erreicht. Obwohl das damit verbundene administrative Verfahren für das Land ein erheblicher Kraftakt war, steht allen Beteiligten, dem Land wie den Kommunen, die größte Herausforderung - die jährliche Aufbringung der Finanzierungsmittel über einen sehr langen Zeitraum - noch bevor. Vor diesem Hintergrund sind die aktuellen Pläne des Bundes zur Lösung der kommunalen Altschuldenproblematik auch für das Land Hessen und seine Kommunen von enormer Bedeutung. Ein sachlicher Grund, Hessen von etwaigen Hilfen auszuschließen, ist nicht erkennbar. Die kommenden Jahre werden zeigen, ob das strategische Ziel der HESSENKASSE auch tatsächlich erreicht wird. Dabei wird es entscheidend sein, auch in wirtschaftlich schlechteren Zeiten, das Ziel nicht aus den Augen zu verlieren. Zweifellos werden dabei auch Entscheidungen zu treffen sein, die sich nicht nur einnahmenseitig, sondern ebenso auch ausgabenseitig auswirken müssen.

$23 \mathrm{Vgl}$. Brand/Steinbrecher (2018), S. 1. 


\section{Literatur}

Boettcher, F. (2012): Kommunale Haushaltsdefizite: Umfang, Ursachen und Lösungsmöglichkeiten, in: dms - der moderne staat - Zeitschrift für Public Policy, Recht und Management, $1 / 2012$, S. 65-84.

Brand, S./Steinbrecher, J. (2018): Kommunales Altschuldenproblem: Abbau der Kassenkredite ist nur ein Teil der Lösung, KfW Research - Fokus Volkswirtschaft Nr. 203 2018. https://www.kfw.de/PDF/Download-Center/ Konzernthemen/Research/PDF-DokumenteFokus-Volkswirtschaft/Fokus-2018/Fokus-Nr.203-April-2018-Altschulden.pdf (09.10.2019)

Bundesministerium der Finanzen: Eckdaten zur Entwicklung und Struktur der Kommunalfinanzen 2009-2018, https://www.bundesfinanzministerium.de/Web/DE/ Themen/Oeffentliche_Finanzen/Foederale_ Finanzbeziehungen/Kommunalfinanzen/kommunalfinanzen.html (og.10.2019)

Gesetz zur Sicherstellung der finanziellen Leistungsfähigkeit der hessischen Kommunen bei liquiditätswirksamen Vorgängen und zur Förderung von Investitionen (HessenkasseG) vom 25. April 2018, GVBI. S. 59-67.

Gesetzentwurf der Fraktionen der CDU und BÜNDNIS 90/DIE GRÜNEN für ein Gesetz zur Sicherstellung der finanziellen Leistungsfähigkeit der hessischen Kommunen bei liquiditätswirksamen Vorgängen und zur Förderung von Investitionen (HessenkasseG), LT-Drs. 19/5957 vom 23.01.2018.

Gesetzentwurf der Fraktionen der CDU und der FDP für ein Gesetz zur Änderung der Hessischen Gemeindeordnung und anderer Gesetze, LT-Drs. 18/4031 vom 10.05.2011.

Gröpl, C./Heinemann, F./Kalb, A. (2010): Die Zweckentfremdung des kommunalen Kassenkredits - eine rechtlich-ökonomische Analyse, Perspektiven der Wirtschaftspolitik 11/2010, S. 178-203.

Heppke-Falk, K./Wolff, G. (2007): Moral hazard and bail-out in fiscal federations: evidence for the German Länder, Deutsche Bundesbank, Discussion Paper Series 1: Economic Studies No. 07/2007.

Herrmann, K. (2011): Der Missbrauch kommunaler Kassenkredite, Wirtschaftsdienst 10/2011, S. 686-693.

Keilmann, U./Duve, T./Gnädinger, M. (2013): Praxisbericht zur Umsetzung des Kommunalen Schutzschirmes in Hessen, in: Verwaltung \& Management, Heft 3, S. 115-122.

Räth, N. (2009): Rezessionen in historischer Betrachtung, Statistisches Bundesamt, Wirtschaft und Statistik 3/2009, S. 203-208.

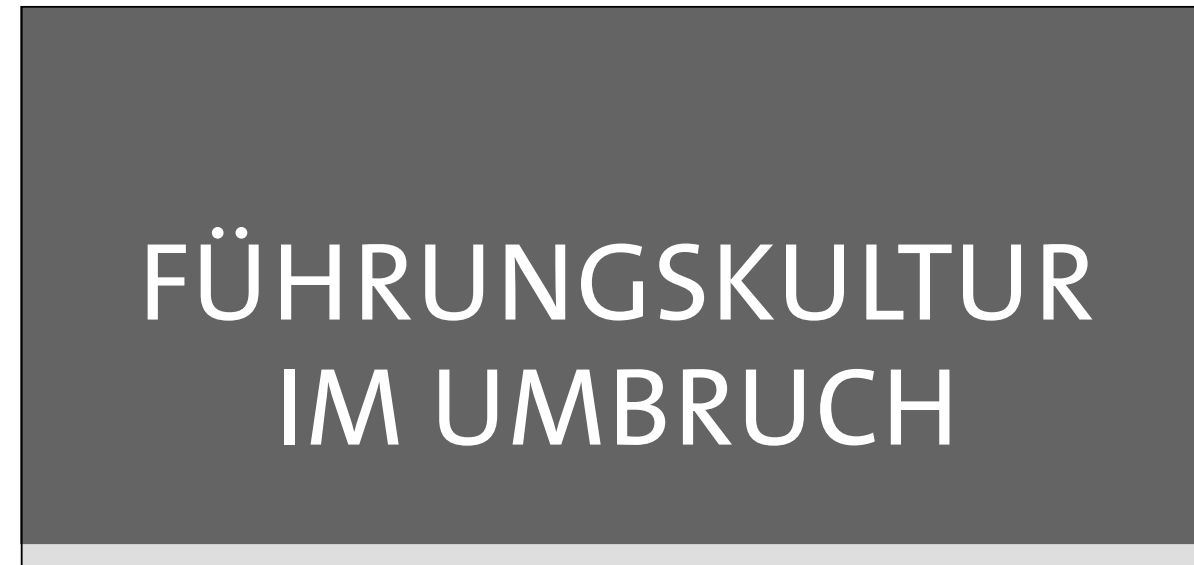

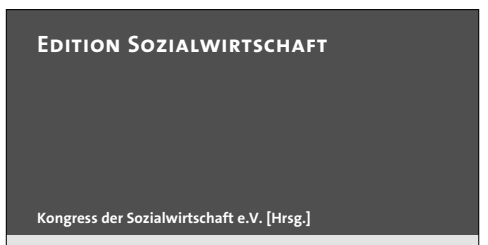

Führung gestaltet

Generationenwechsel - Digitalisierung - Kulturwandel Bericht über den 11. Kongress der Sozialwirtschaft vom 16. und 17. Mai 2019 in Magdeburg (1)

Nomos

\section{Führung gestaltet}

Generationenwechsel Digitalisierung - Kulturwandel Bericht über den 11. Kongress der Sozialwirtschaft vom 16. und 17. Mai 2019 in Magdeburg

Herausgegeben vom Kongress der Sozialwirtschaft e. V.

2019, 234 S., brosch., 49,- $€$

ISBN 978-3-8487-6252-1

(Edition Sozialwirtschaft, Bd. 45)

nomos-shop.de/43375
„Was erwarte ich von einer modernen Führungskultur?“, lautete die zentrale Frage des Generationendialogs auf dem Kongress der Sozialwirtschaft 2019. Generationenwechsel, Digitalisierung und Kulturwandel stellen nicht nur Unternehmen der Sozial- und Gesundheitswirtschaft vor eine Bewährungsprobe: Die Arbeitswelt im Allgemeinen dynamisiert sich, tradierte Geschäftsmodelle und Strukturen stehen vor Transformationsprozessen und disruptive Entwicklungen lösen normale Erneuerungsphasen ab.

Der vorliegende Tagungsband beleuchtet u. a. innovative Denkansätze, agile Strukturen, Führung ohne Hierarchie, Diversity Management, die Führungskraft der Zukunft und eine gesunde Unternehmenskultur. Denn die Zeit der kontinuierlichen Veränderungen ist vorbei, eine Zeit des Umbruchs hat begonnen.

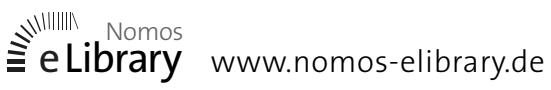

Erhältlich im Buchhandel oder versandkostenfrei unter www.nomos-shop.de Alle Preise inkl. Mehrwertsteuer 